\title{
The multiple V-shaped double peeling of elastic thin films from elastic soft substrates.
}

\author{
N. Menga ${ }^{1}$ L. Afferrante, ${ }^{1, *}$ N. M. Pugno, ${ }^{2,3,4}$ and G. Carbone ${ }^{1,5,6}$ \\ ${ }^{1}$ Department of Mechanics, Mathematics and Management, \\ Politecnico of Bari, V.le Japigia, 182, 70126, Bari, Italy \\ ${ }^{2}$ Laboratory of Bio-Inspired and Graphene Nanomechanics, \\ Department of Civil, Environmental and Mechanical Engineering, \\ University of Trento, Via Mesiano 77, 38123 Trento, Italy \\ ${ }^{3}$ School of Engineering and Materials Science, \\ Queen Mary University of London, \\ Mile End Road, London E1 4NS, United Kingdom \\ ${ }^{4}$ Ket Lab, Edoardo Amaldi Foudation, Italian Space Agency, \\ Via del Politecnico snc, 00133 Rome, Italy \\ ${ }^{5}$ CNR - Institute for Photonics and Nanotechnologies U.O.S. Bari, \\ Physics Department "M. Merlin", via Amendola 173, 70126 Bari, Italy \\ ${ }^{6}$ Imperial College London, Department of Mechanical Engineering, \\ Exhibition Road, London SWr $2 A Z$
}

\begin{abstract}
In this paper, a periodic configuration of $\mathrm{V}$-shaped double peeling process is investigated. Specifically, an elastic thin film is detached from a soft elastic material by applying multiple concentrated loads periodically distributed with spatial periodicity $\lambda$. The original Kendall's idea is extended to take into account the change in elastic energy occurring in the substrate when the detachment fronts propagate. The symmetric configuration typical of a $\mathrm{V}$-peeling process causes the energy release rate to be sensitive to the variations of elastic energy stored in the soft substrate. This results in an enhancement of the adhesion strength, because part of the external work required to trigger the peeling mechanism is converted in substrate elastic energy.

A key role is played by both spatial periodicity $\lambda$ and elasticity ratio $E / E_{h}$, between tape and substrate elastic moduli, in determining the conditions of stable adhesion. Indeed, the presence of multiple peeling fronts determines a modification of the mechanism of interaction, because deformations close to each peeling front are also affected by the stresses related to the other fronts. Results show that the energy release rate depends on the detached length of the tape so that conditions can be established which lead to an increase of the supported load compared to the classical peeling on rigid substrates.

Finally, we also find that for any given value of the load per unit length, an optimum value of the wavelength $\lambda$ exists that maximizes the tolerance of the system, before unstable propagation of the peeling front can occur.
\end{abstract}

Keywords: V-shaped double peeling, adhesion, energy release rate, pressure sensitive adhesives.

*Electronic address: [Correspondingauthor.] Email:luciano.afferrante@poliba.it, phonenumber: $+390805962704$ 


\section{INTRODUCTION}

In the last decades, biological and biomechanical applications have boosted the interest in the adhesive contact of thin films, making it an important topic in contact mechanics [111]. In nature, several organisms show enhanced adhesion thanks to hierarchical structures of hairs or setae. For instance, hairy attachment systems of insects, arachnids and reptiles have been intensively studied during the past years, aiming at explaining and possibly mimic their extraordinary adhesive abilities in artificial bio-mimetic devices [12-21].

To this regard, several studies have been devoted to soft elastic contacts in presence of adhesion [11, 22-25], and specifically to the detachment process [26-28]. Experimental observations of insects and spiders [29-31] and theoretical studies [32-34] have made clear the crucial role played by highly flexible terminal spatula-shaped substructures attached to their legs, which finally allows them to easily climb on surfaces with different properties in terms of roughness and compliance.

The role of roughness is not yet completely understood. Several theoretical [35-37], numerical [24, 38-40] and experimental [41-44] studies have shown that high surface roughness reduces interfacial adhesion. However, some insects, like geckos, are able to achieve extremely high adhesive performance also on rough substrates. Some models have been proposed which attempt to explain this interesting behavior [6, 23, 31, 45, 46]. For instance, in Ref. [46], it is shown that high adhesion forces are obtained by rolling down and inward the toes to realize small peeling angle during the attachment. Detachment is instead obtained by rolling the toes upward and backward. Also, the gecko ability to remain stuck while inverted on the ceiling has been investigated in Refs. [47, 48]. Those studies show that geckos use opposing feet and toes to prevent detachment of setae or peeling of toes.

Therefore, since the 50's, several studies have been devoted to understand the mechanism governing peeling [49-52]. Interest in practical applications, such as defining specific peeling tests standards to characterize the adhesive properties of joints involving thin films [53, 54], has led researchers to focus on phenomena involved in peeling process. In this respect, a very comprehensive review is provided in Ref. [55]. About phenomena occurring at interface, several works have investigated the intrinsic bi-layered nature of typical commercial tapes (e.g. 3M Scotch), where the adhesive soft layer is backed to a thin stiffer one. In this case, during the peeling process a loss of confinement of the soft adhesive part gives rise to generation of a macroscopic debonding region characterized by fibrillar adhesive bridges and progressive bending deformation of the tape [56-58]. It is also interesting to recall that when the adhering surfaces are rough, air pockets can remain trapped at the interface, depending on the material properties, the strength of adhesive interactions, and the topography of the surface (see, for example, Refs. [59-61]). Moreover, in some cases, as the peeling process advances and the interface between the layers is interested by tensile stress, high speed camera images (see Ref. [62]) show nucleation and growth of cavities, near to the peak stress region, which make the layers deformation no longer homogeneous.

Such studies typically rely on the assumption of compliant films on rigid substrates. For a large class of practical applications, this hypothesis leads to physically realistic results. However, there exists several other applications, where a more proper formalism is required to take into account the effect of the substrate compliance on the detachment mechanism, as for instance studying the adhesive contact of medical Band-aids on human skin [63, 64]. In this case, indeed, the deformability of the substrate have to be taken into account, and although the skin is usually modeled as a very compliant viscoelastic material [65-68], interesting 
results may be inferred neglecting viscous effects at low speeds, because in such case the behavior is essentially elastic [69-71].

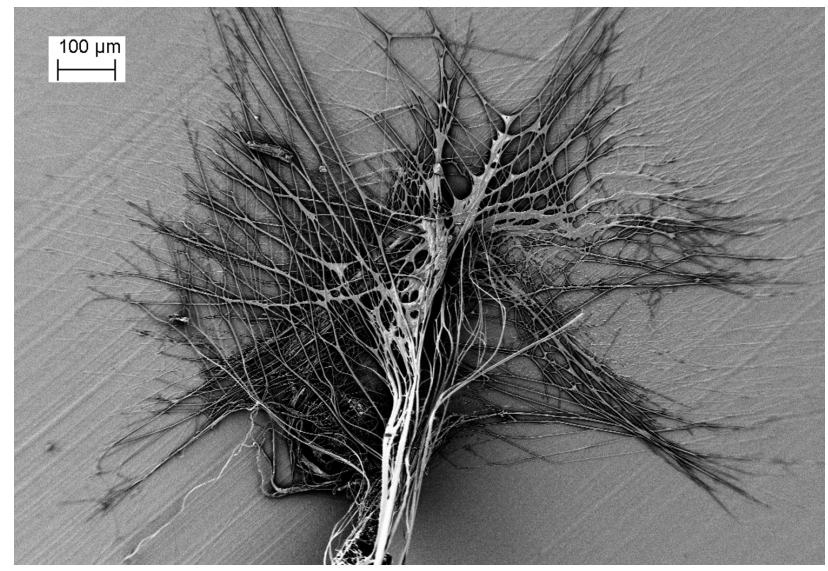

FIG. 1: A Scanning electron microscope image of a spider web anchor. Multiple V-shaped attachment points can be seen.

Moreover, dealing with deformable substrates, the specific geometrical configuration of the peeling system strongly affects the process evolution. As reported in Refs. [7, 72, 73], specific patterns of multiple and opposite peeling fronts can be recognized in the detachment process of gecko's toes as well as in proximity of spider webs anchors (see Fig. 1). In these systems, the interaction between the elastic fields, generated by the multiple peeling fronts, plays a key role in determining the detachment behavior. Indeed, differently from the classical Kendall's peeling, where the mechanism of detachment is unaffected by the elastic properties of the substrate $[51,74]$, in the $\mathrm{V}$-shaped double peeling case translation invariance is lost due to the symmetry of the problem. Consequently, as the peeling front advances, variation in elastic energy stored in the substrate cannot be neglected. This entails a change in the external work required to trigger the detachment process, which in turn modifies the peeling mechanism.

We stress that for the first time it is made clear that the combined effect of considering a V-shaped peeling configuration (which causes the loss of translation invariance) and a compliant substrate (which makes possible interaction between the elastic displacements fields associated with the peeling fronts) results in an enhancement of the adhesion strength.

\section{FORMULATION}

Consider the symmetric V-shaped periodic double peeling scheme shown in Fig. 2. The system is constituted by an elastic thin film of thickness $d$, and transversal width $b$, adhering to a linear elastic half-plane. The film is assumed infinitely flexible, and is peeled off by applying multiple vertical forces $2 P$ periodically distributed with spatial periodicity $\lambda$.

Under the assumption of linearity and small deformations, physical arguments (see Ref. [74]) suggest that the overall load $T=P /(\sin \theta)$ distributes in proximity of the detachment front over a strip of length $2 a$ of the same order of $d$. Consequently, the surface displacements of the elastic substrate can be calculated with good accuracy by considering two uniform distributions of normal $p_{n}(x)=p=P /(2 a b)$ and tangential $p_{t}(x)=q=-P \cot \theta /(2 a b)$ tractions, acting on a strip of size $2 a \simeq \alpha d$, where $\alpha$ is a proportionality constant expected 

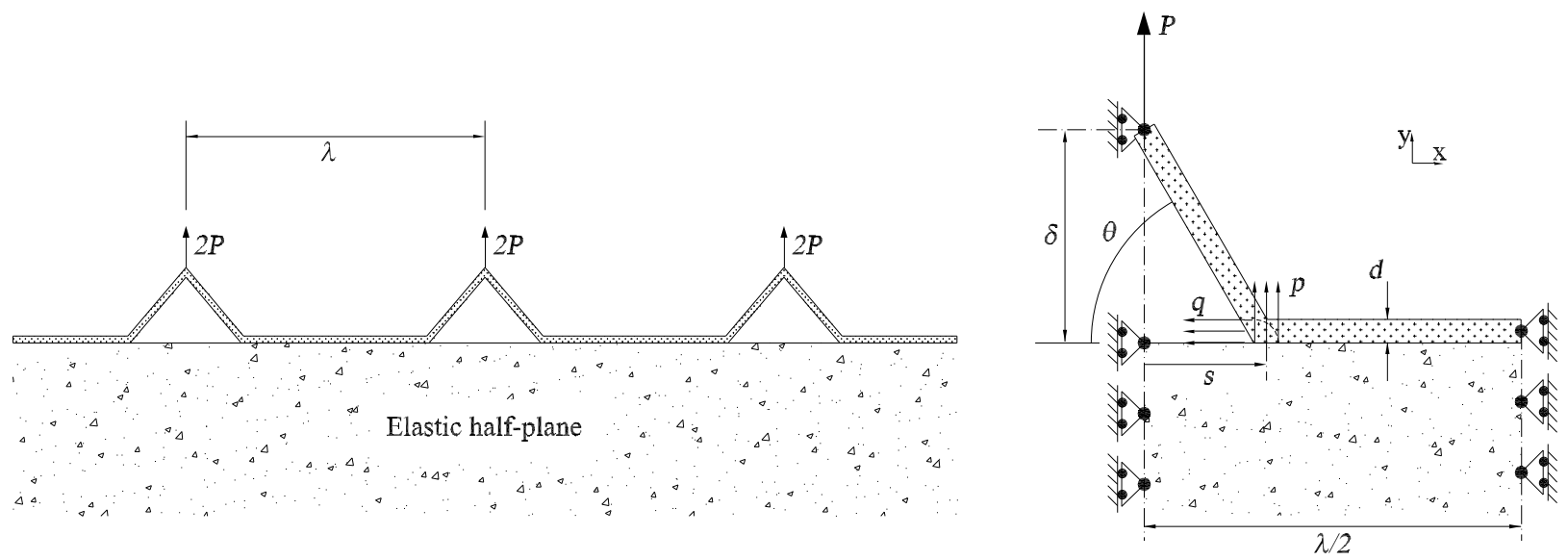

FIG. 2: The periodic V-shaped double peeling scheme. Periodicity and symmetry of the problem can be exploited to focus the study on half of a single elementary cell.

to be of the order of the unity; thus for the following numerical examples we have fixed $\alpha=1$. With reference to normal stresses, such assumption is indeed confirmed in Ref. [75]; about shear stresses, one can easily show that they are necessarily distributed on a region of size proportional to $\left(E / E_{h}\right)^{1 / 2} \sqrt{\lambda d}$, being $E$ and $E_{h}$ the elastic moduli of the thin film and half-plane,respectively. However, though our assumption about the extension of the region of distribution of tangential tractions may be a bit stronger in some cases, we expect it does not modify qualitatively the main physical scenario.

The symmetry typical of a double V-peeling configuration entails the loss of translation invariance, and makes the calculation of the energy release rate sensitive to the variation of the elastic energy in the substrate during the peeling fronts propagation. Such energy variation is also affected by the interaction between the peeling fronts, as deformations close to a peeling front also depend on the stresses related to the other fronts. In particular, with reference to Fig. 2 in order to calculate the surface displacements of the substrate, two mechanisms of interaction need to be considered. The first one is related to the fronts belonging to the same $\mathrm{V}$-peel, and is taken into account by superposing the displacements due to each single peeling front: $\mathbf{u}^{s}(s, x)=\mathbf{u}(x-s)+\mathbf{u}(x+s)$, being $s$ the detached length. The latter is related to the periodic adjacent V-peels, and is intrinsically considered by calculating the displacements vector $\mathbf{u}=\left(u_{x}, u_{y}\right)$, due to the periodic surface tractions $q$ and $p$, by means of the Green's functions given in appendix A.

In presence of very soft materials, large deformations might magnify the effects of interaction between peeling fronts, but do not modify the main physics governing the considered problem, i.e. the qualitative behavior of the peeling process is not affected by the presence of large deformations. Indeed, under the JKR assumption [76], large deformations and material nonlinearity have small effects on the adhesion of soft elastic materials (see, for example, Ref. [77]).

In the framework of the above assumptions, it is straightforward demonstrating that the continuity equation writes as

$$
\delta \sin \theta=s(1-\cos \theta+\varepsilon)
$$

where $\delta \sin \theta$ is the component of the displacement of the point of application of $P$ along the tape direction and $\varepsilon=\left(\mathrm{d} l^{\prime}-\mathrm{d} l\right) / \mathrm{d} l=P /(E b d \sin \theta)$ is the elongation of the tape per 
unit length, being $E$ the Young's modulus of the tape material. Furthermore, from simple geometrical considerations we can also write

$$
s(1+\varepsilon) \cos \theta=s \quad \rightarrow \quad\left(1+\frac{P}{E b d \sin \theta}\right) \cos \theta=1
$$

which defines a one to one relation between the applied force $P$ and the peeling angle $\theta$. Moreover, to completely define the problem we need to find the equilibrium condition. For a reversible and isothermal transformation, the system spontaneously moves out of equilibrium when the variation of the total energy is negative, i.e.

$$
\mathrm{d} U_{t o t}=\mathrm{d} U_{e l, t}+\mathrm{d} U_{e l, h}+\mathrm{d} U_{P}+\mathrm{d} U_{a d}<0
$$

where $\mathrm{d} U_{e l, t}$ and $\mathrm{d} U_{e l, h}$ are, respectively, the change in the elastic energy stored in the tape and substrate, $\mathrm{d} U_{P}$ is the variation of the potential energy associated with the applied peeling force $P$ and $\mathrm{d} U_{a d}$ is the change in the surface energy. The surface adhesion energy is $U_{a d}=\Delta \gamma b s$, being $\Delta \gamma$ the Duprè energy of adhesion, whereas the elastic strain energy stored in the tape can be calculated as $U_{e l, t}=T^{2} s /(2 E b d)$. Considering that the bending stiffness of the tape depends on the cubic power of thickness $d$, the contribution of bending in terms of change of elastic energy during peeling propagation is negligible, as indeed observed in Ref. [57]. The elastic energy stored in the half-plane is

$$
U_{e l, h}=\frac{1}{2} b \int_{s-d / 2}^{s+d / 2} \sigma \cdot \mathbf{u}_{t o t}(s, x) \mathrm{d} x
$$

where $\sigma=(q, p)$ is the vector of the normal and tangential tractions. The potential energy is instead $U_{P}=-P u_{P}$, where $u_{P}$ is the total vertical displacement of the point of application of $P$

$$
u_{P}=u_{y, t o t}(s, s)+\delta=u_{y, t o t}(s, s)+s \tan \theta
$$

Recalling that the energy release rate $G$ on the peeling front is defined as (Ref. [78])

$$
G=-\frac{\partial\left(U_{t o t}-U_{a d}\right)}{\partial A}=-\frac{1}{b}\left(\frac{\partial U_{e l, t}}{\partial s}+\frac{\partial U_{e l, h}}{\partial s}+\frac{\partial U_{P}}{\partial s}\right)
$$

eq. (3) can be rewritten as

$$
\mathrm{d} U_{t o t}=-(G-\Delta \gamma) b \mathrm{~d} s<0
$$

Therefore, if $G>\Delta \gamma$ the above inequality requires $\mathrm{d} s>0$ and the crack will spontaneously advance causing the detachment of the tape; if $G<\Delta \gamma$, then $\mathrm{d} s<0$ and peeling is prevented. As a result, the equilibrium condition can be obtained by enforcing $G=\Delta \gamma$. The above formulation has been obtained under the assumption of negligible inertia effects. As a consequence, our results are expected to be physically sound for peeling velocities sufficiently smaller than the characteristic propagation speed of waves in the material. Under dynamic conditions, both experimental and numerical investigations $[52,79,80]$ show the occurrence of dynamic instabilities, which cannot be captured with the present model. In such a case, in particular, the calculation of the energy release rate should also take into account the kinetic energy contribution [81, 82]. 


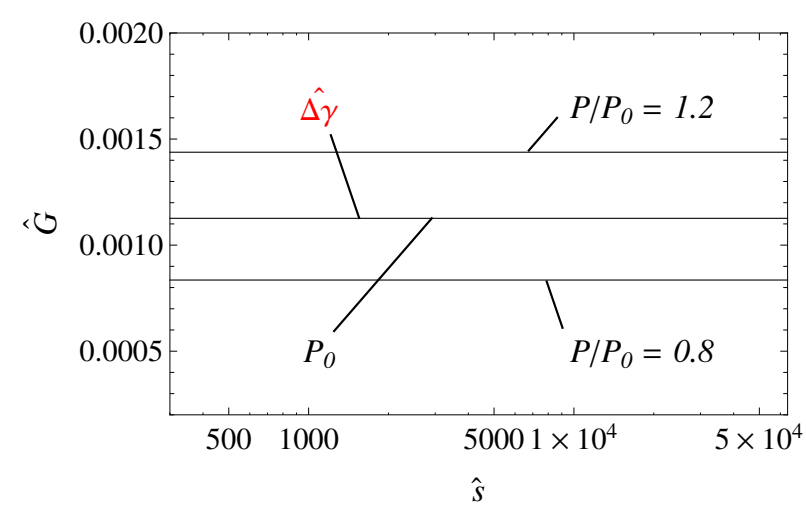

(a)

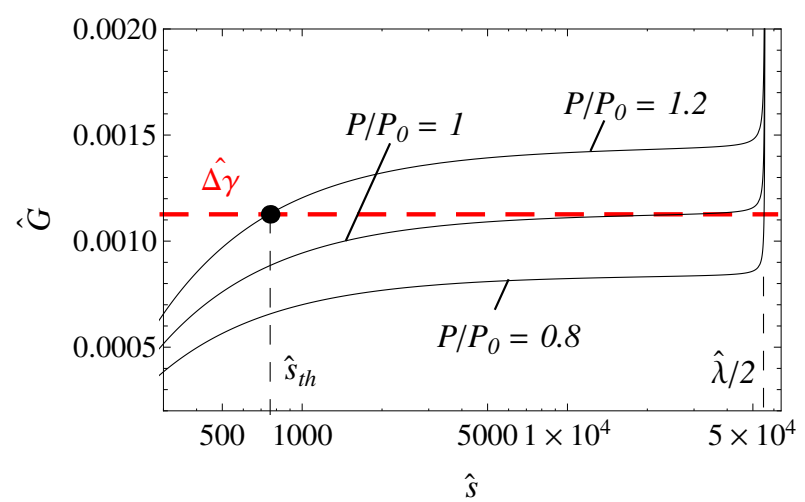

(b)

FIG. 3: The dimensionless energy realease rate $\hat{G}$ as a function of the dimensionless detached length $\hat{s}$, for different applied loads $P / P_{0}$ on rigid (a) and compliant (b) substrate, respectively. The value of the dimensionless work of adhesion $\Delta \hat{\gamma}=1.126$ is plotted with red dashed line. Results are obtained for $\chi=E / E_{h}=500$ and $\lambda / d=10^{5}$. The values on $\mathrm{x}$-axis are given in log scale.

\section{RESULTS}

Results are given for substrate of incompressible material (Poisson's ratio $\nu_{h}=0.5$ ).

Figure 3 shows, in a semi-log plot, the dimensionless energy release rate $\hat{G}=G / E d$ as a function of the dimensionless detached length $\hat{s}=s / d$, at different applied loads, for rigid (3a) and compliant (3b) substrate, respectively. The dimensionless work of adhesion $\Delta \hat{\gamma}=\Delta \gamma /(E d)$ is plotted with red dashed line. In particular, in Fig. 3a, the solution given in $[83,84]$ is plotted for tape initially fully stuck to the substrate $(h \rightarrow 0$, being $h$ a length of the tape initially not attached). When the substrate is rigid, $\hat{G}$ is not affected by the detached length. Moreover, peeling is prevented when $P / P_{0}<1$ and unstable detachment always occurs for $P / P_{0}>1$, being $P_{0}$ the load value for which $\hat{G}=\Delta \hat{\gamma}$. Notice $P_{0}=T_{0} \sin \theta$, where $T_{0}$ corresponds to the Kendall's critical load, which can be obtained by solving

$$
\frac{T_{0}}{b}\left(1-\cos \theta+\frac{T_{0}}{E b d}\right)=\Delta \gamma
$$

A different behavior is observed in Fig. 3b for compliant substrate. First, $\hat{G}$ monotonically increases with $\hat{s}$. Therefore, stable adhesion is possible even for $P / P_{0}>1$ provided the detached length $s$ is lower than a threshold value $s_{t h}(P)$, which depends on the load. Above $s_{t h}, G$ becomes larger than $\Delta \gamma$, and unstable propagation of the peeling front is expected to occur.

According to the Griffith's criterion, equilibrium occurs at the point $\hat{G}=\Delta \hat{\gamma}$. However, such point of equilibrium is unstable. In fact, $\Delta \gamma$ represents the work required to advance the detachment front by one unit length, and $G$ the energy available for the peeling front propagation. Therefore, starting from the equilibrium, a small increment of the detached length involves $G>\Delta \gamma$, i.e. there is more released energy than the adhesion energy gained by separating the surface, leading to peeling propagation instability. Figure $3 \mathrm{~b}$ also shows 
that for a periodic $\mathrm{V}$-shaped peeling, the tape can sustain loads larger than $P_{0}$ provided that $s<s_{t h}(P)$.

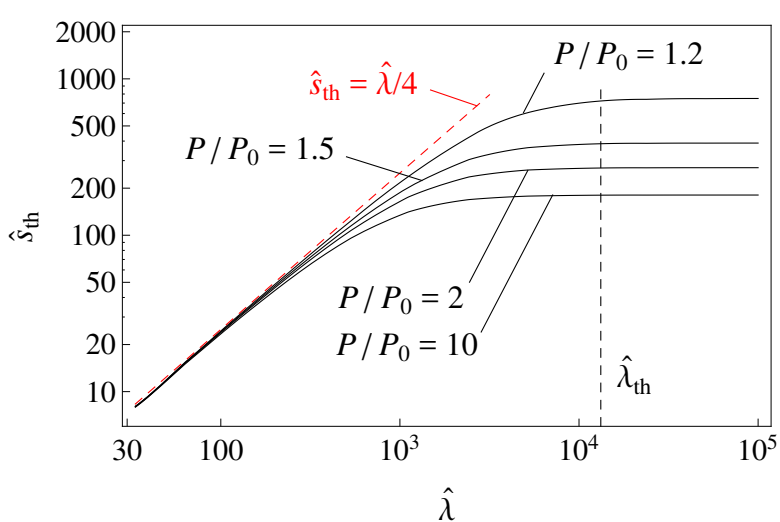

(a)

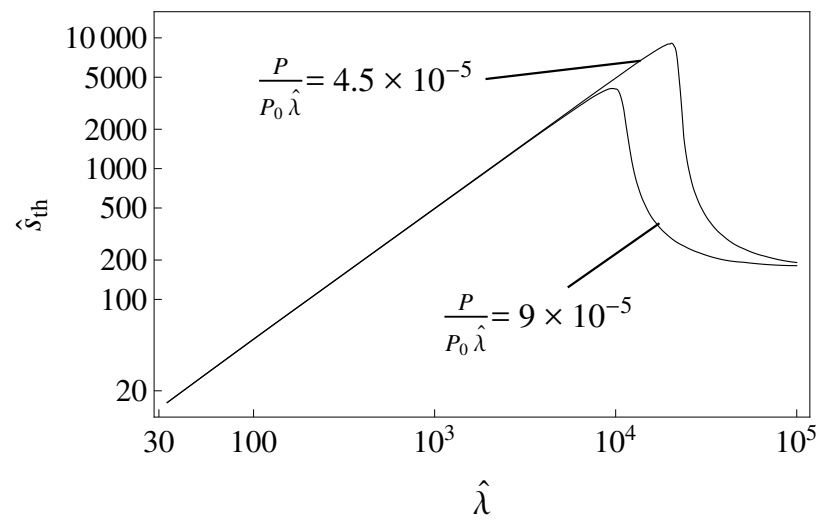

(b)

FIG. 4: The dimensionless threshold value $\hat{s}_{t h}$ of the detached length as a function of the dimensionless load pitch $\hat{\lambda}$ (a) for different applied loads, (b) for different loads per unit length. Results are given for $\chi=E / E_{h}=1000$ and $\Delta \hat{\gamma}=1.126$.

Fig. 4a shows the dimensionless threshold value $\hat{s}_{t h}=s_{t h} / d$ of the detached length as a function of the dimensionless spatial periodicity $\hat{\lambda}=\lambda / d$, for different applied loads. We note that, almost independently of the applied load, the quantity $s_{t h}$ is proportional to the spatial periodicity $\lambda$, provided that $\lambda$ is not too large, taking the value $s_{t h}=\lambda / 4$, which corresponds to a distance between the two peeling fronts just equals half the spatial periodicity, i.e. to a configuration where all the peeling fronts are equi-spaced. On the other hand for sufficiently large value of $\lambda$ the threshold value $s_{t h}$ reaches a plateau, which instead significantly depends on the applied load $P$.

Further, interesting conclusions can be drawn from Fig. 4b, where, this time, the relation between $\hat{s}_{t h}$ and $\hat{\lambda}$ is investigated at fixed load per unit length. Specifically, $s_{t h}$ linearly increases with $\lambda$ up to a critical value beyond which a strong reduction is observed. This behavior, peculiar of periodic $\mathrm{V}$-shaped double peeling processes, is of straightforward interest for engineering applications, entailing the possibility to arrange the contacts with the specific spatial periodicity $\lambda$ that, given the size of the initial defect (i.e., the initial detached length), maximizes the peeling load per unit length.

\section{A. The asymptotic limit $\lambda \rightarrow \infty$}

In the limit of large values of $\lambda$, the asymptotic aperiodic behavior corresponding to the geometric scheme of Fig. 5 is recovered.

For this case, Fig. 6 shows the variation of the threshold $\hat{s}_{t h}$ with the peeling load $P$, normalized with respect to $P_{0}$, for different values of the elasticity ratio $\chi=E / E_{h}$ between the tape and half-space elastic moduli.

Increasing $P / P_{0}$ leads to an initial sharp reduction in $\hat{s}_{t h}$, which then converges to a limiting value at high loads. In particular, if the elasticity ratio $\chi$ is not too large, for loads $P$ just exceeding $P_{0}$, the threshold $\hat{s}_{t h}$ takes small values and adhesion can be guaranteed only 

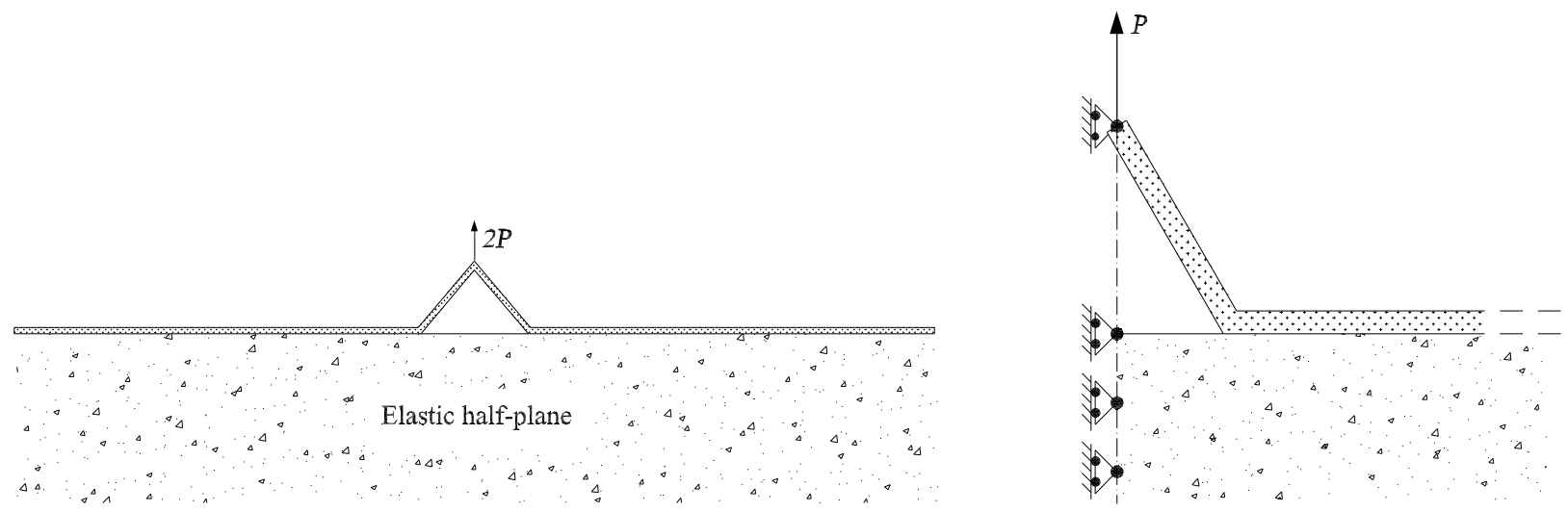

FIG. 5: The scheme of single V-shaped double peeling of an elastic thin film from a compliant elastic substrate.

under complete contact conditions. However, in such a case, even a small perturbation at the peeling front with a slight increase in $s$ would be sufficient to cause unstable detachment. On the contrary, when $\chi$ is high the decrease of $\hat{s}_{t h}$ with $P / P_{0}$ is less sharp. In such a case, given the load and starting from complete contact, initial small detachments could be tolerated without unstable propagation of the peeling front taking place. Notice the area below the curves corresponds to the region of stable adhesion, whereas above the curves unstable detachment is expected occurring.

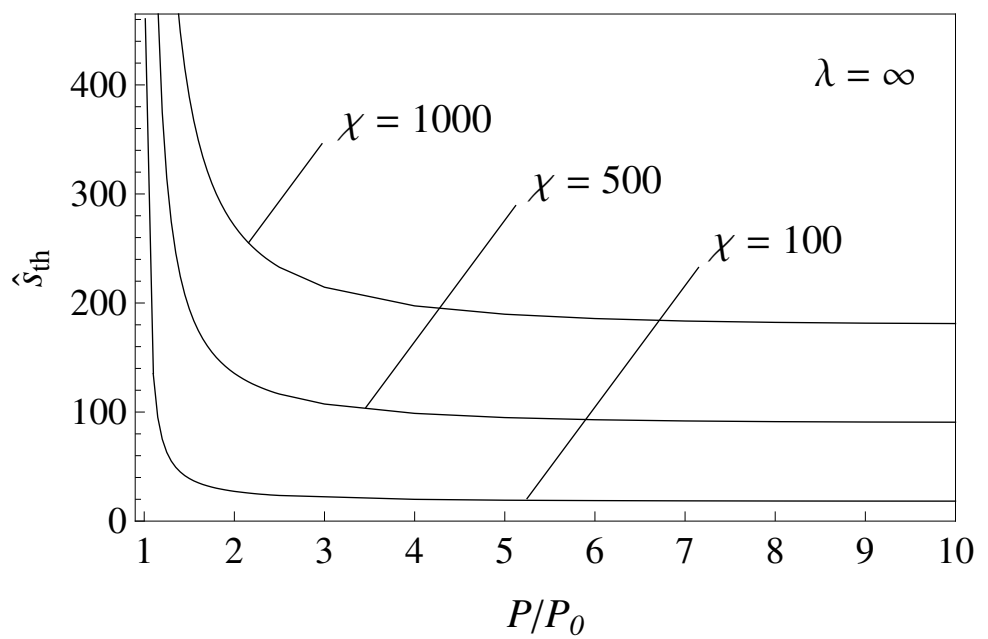

FIG. 6: The dimensionless threshold of the detached length $s_{t h}$ as a function of the normalized peeling load $P / P_{0}$ for different values of the elasticity ratio $\chi=E / E_{h}$. The regions under the curves correspond to stable and safe states of adhesion. Above the curves unstable detachment is expected.

These arguments, which are also valid for the periodic case, suggest that, in principle, we could tune the value of the elasticity ratio $\chi$ to make sufficiently high the threshold $s_{t h}$ and, as a result, to enhance the adhesive strength of the system. 


\section{CONCLUSIONS}

In this paper, a mathematical model to study the periodic V-shaped double peeling of elastic films from soft substrates is proposed in the framework of linear elasticity. Specifically, the effect of substrate's compliance and periodically distributed multiple loads on the mechanism of peeling is investigated. An increase in the adhesion strength is found with respect to the case of rigid substrate. Such increase is due to the symmetry characterizing the geometrical configuration of the double $\mathrm{V}$-peeling that determines the loss of translation invariance, which in turn makes the calculation of $G$ sensitive to the variation of the elastic energy in the substrate.

Results show that the adhesion strength is significantly affected by the elasticity ratio $\chi$ between the Young's moduli of tape and substrate and the spatial periodicity $\lambda$. In particular, an increase in both $\chi$ and $\lambda$ produces a strong enhancement of the defect tolerance of the system, i.e. initially detached lengths can be easily tolerated without causing peeling propagation and then detachment. Moreover, given the size of the initial 'defect', it is possible to arrange the contacts, by choosing the proper value of $\lambda$, in order to maximize the load per unit length which can be borne by the tape.

Acknowledgement 1 The authors want to thank Gabriele Greco who took image of Fig. 1. N. M. P. is supported by the European Commission H2020 under Graphene Flagship Core 1 No. 696656 (WP14 "Polymer composites") and FET Proactive "Neurofibres" Grant No. 732344.

\section{APPENDIX A: ELASTIC SURFACE DISPLACEMENTS DUE TO PERIODIC UNIFORM NORMAL AND TANGENTIAL STRESS DISTRIBUTIONS}

Consider a uniform distribution of normal $p$ and tangential $q$ tractions acting on an elastic half-plane over a strip of size $2 a=d$. Assume such tractions are periodically distributed with periodicity $\lambda$. According to Ref. [24], the total surface displacement vector $\mathbf{v}=\left(v_{x}, v_{y}\right)$ can be written as the convolution product between the Green's tensor $\mathbf{G}(x)$ and the interfacial stress vector $\sigma=(q, p)$

$$
\mathbf{v}(x)-\mathbf{v}_{m}=\int_{\Omega} d s \mathbf{G}(x-s) \sigma(s) ; \quad x \in[0, \lambda]
$$

where $\Omega$ is the domain where the surface stresses are applied, $\mathbf{v}_{m}$ is the mean displacement vector and the components of the the Green's tensor are given by ([24])

$$
\begin{aligned}
& G_{11}(x)=-\frac{2\left(1-\nu_{h}^{2}\right)}{\pi E_{h}} \log \left|2 \sin \left(\frac{k x}{2}\right)\right| \\
& G_{21}(x)=-\frac{1+\nu_{h}}{\pi E_{h}} \frac{1-2 \nu_{h}}{2}[\operatorname{sgn}(x) \pi-k x] \\
& G_{22}(x)=-\frac{2\left(1-\nu_{h}^{2}\right)}{\pi E_{h}} \log \left|2 \sin \left(\frac{k x}{2}\right)\right|
\end{aligned}
$$

where $E_{h}$ and $\nu_{h}$ are, respectively, the Young's modulus and the Poisson's ratio of the substrate material and $k=2 \pi / \lambda$. Substituting the above relations in (A1) an after same 
algebraic applications, the effective normal $u_{y}=v_{y}-v_{m, y}$ and tangential $u_{x}=v_{x}-v_{m, x}$ surface displacements can be written as

$$
\begin{aligned}
& u_{y}(x)=F_{1}(x) p-F_{2}(x) q \\
& u_{x}(x)=F_{1}(x) q+F_{2}(x) p
\end{aligned}
$$

where the functions $F_{1}(x)$ and $F_{2}(x)$ are so defined

$$
\begin{aligned}
& F_{1}(x)=-\frac{2\left(1-\nu_{h}^{2}\right)}{\pi E_{h}}\left[C l_{2}(x-a)-C l_{2}(x+a)\right] \\
& F_{2}(x)=-\frac{\left(1-2 \nu_{h}\right)\left(1+\nu_{h}\right)}{\pi E_{h}}[\Phi(x-a)-\Phi(x+a)]
\end{aligned}
$$

being

$$
C l_{2}(x)=-\int_{0}^{x} \log |2 \sin (t / 2)| d t
$$

the Clausen function of order 2 (Ref. [85]), and

$$
\Phi(x)=\frac{(k x)^{2}}{4}+k x \arctan [\cot (x / 2)]
$$

Finally, the total surface displacements are obtained by superposition of (A5, A6) as $\mathbf{u}^{s}(s, x)=\mathbf{u}(x-s)+\mathbf{u}(x+s)$, being $s$ the distance of the load distributions from the symmetry axis.

[1] Varenberg, M., Pugno, N., Gorb, S., 2010, Spatulate structures in biological fibrillar adhesion. Soft Matter 6, 3269-3272.

[2] Ding, J., Wen, S., Meng, Y. 2001, Theoretical study of the sticking of a membrane strip in MEMs under the Casimir effect. J Micromech Microeng 11, 202-208.

[3] Shanahan, M.E.R., 2000, Adhesion of a punch to a thin membrane. CR Acad Sci Ser IV 1, 517-522.

[4] Wan, K.T., 2001, Adherence of an axisymmetric flat punch on a flexible membrane. J Adhesion $75,369-380$.

[5] Jin, C., 2009, Theoretical study of mechanical behavior of thin circular film adhered to a flat punch. Int J Mech Sci 51 301-313.

[6] Pesika, N.S., Tian, Y., Zhao, B., Rosenberg, K., Zeng, H., McGuiggan, P., Autumn, K., Israelachvili, J.N., 2007, Peel-Zone Model of Tape Peeling Based on the Gecko Adhesive System. J Adhesion 83(4), 383-401.

[7] Pugno, N.M., 2011, The theory of multiple peeling. Int J Fract 171, 185-193.

[8] Carbone, G., Pierro, E. and Gorb, S., 2011, Origin of the superior adhesive performance of mushroom-shaped microstructured surfaces, Soft Matter, 7, 5545-5552.

[9] Afferrante, L. and Carbone, G., 2013, The Mechanisms of Detachment of Mushroom-Shaped Micro-Pillars: From Defect Propagation to Membrane Peeling, Macromol. React. Eng., 7, 609-615. 
[10] Dening K., Heepe L., Afferrante L., Carbone G. and Gorb S. N., 2014, Adhesion control by inflation: implications from biology to artificial attachment device, Appl. Phys. A, 116(2), 567-573.

[11] Menga, N., Afferrante, L. and Carbone, G., 2016, Adhesive and adhesiveless contact mechanics of elastic layers on slightly wavy rigid substrates, Int. J Solids Struct., 88-89, 101-109.

[12] Gravish, N., Wilkinson, M., Autumn, K., 2008, Frictional and elastic energy in gecko adhesive detachment. J R Soc Interface 6, 339-348.

[13] Geim, A. K., Dubonos, S. V., Grigorieva, I. V., Novoselov, K. S., Zhukov, A. A., \& Shapoval, S. Y., 2003, Microfabricated adhesive mimicking gecko foot-hair. Nature materials, 2(7), 461463.

[14] Glassmaker, N.J., Jagota, A., Hui, C.-Y., Noderer, W.L., Chaudhury, M.K., 2007, Biologically inspired crack trapping for enhanced adhesion. Proc. Natl. Acad. Sci. USA 104, 10786-10791.

[15] Pugno, N. M., 2008, Spiderman gloves. Nano Today, 3(5), 35-41.

[16] Krahn, J., Liu, Y., Sadeghi, A., Menon, C. 2011 A tailless timing belt climbing platform utilizing dry adhesives with mushroom caps. Smart Mater. Struct. 20, 115021.

[17] Murphy, M.P., Kute, C., Menguc ,Y., Sitti, M. 2011 Waalbot II: adhesion recovery and improved performance of a climbing robot using fibrillar adhesives. Int. J. Robot. Res. 30, 118-133.

[18] Afferrante, L. and Carbone, C., 2012 Biomimetic surfaces with controlled direction-dependent adhesion, J. R. Soc. Interface, 9, 3359-3365.

[19] Afferrante, L., Grimaldi, G., Demelio, G. and Carbone, G., 2015, Direction-dependent adhesion of micro-walls based biomimetic adhesives, Int. J. Adhesion \& Adhesives, 61, 93-98.

[20] Carbone, G., \& Pierro, E. (2012). Effect of interfacial air entrapment on the adhesion of bio-inspired mushroom-shaped micro-pillars. Soft Matter, 8(30), 7904-7908.

[21] Carbone, G., \& Pierro, E. (2012). Sticky Bio-inspired Micropillars: Finding the Best Shape. Small, 8(9), 1449-1454.

[22] Hui, C.Y., Lin, Y.Y., aney, J.M. \& Kramer, J.E., 2001, The Mechanics of Contact and Adhesion of Periodically Rough Surfaces, Journal of Polymer Science Part B: Polymer Physics, 39(11), 1195-1214.

[23] Persson, B. N. J., 2003, On the mechanism of adhesion in biological systems, J. Chem. Phys., 118, 7614-7621.

[24] Carbone G., Mangialardi L., 2008, Analysis of adhesive contact of confined layers by using a Green's function approach, The Journal of the Mechanics and Physics of Solids, 56 (2), 684-706.

[25] Menga, N., Carbone, G., \& Dini, D. (2018). Do uniform tangential interfacial stresses enhance adhesion?. Journal of the Mechanics and Physics of Solids, 112, 145-156.

[26] Lee, J., Fearing, R. S., \& Komvopoulos, K., 2008, Directional adhesion of gecko-inspired angled microfiber arrays. Applied Physics Letters, 93(19), 191910.

[27] del Campo A. ,Greiner C. ,Artz E., 2007, Contact shape controls adhesion of bioinspired fibrillar surfaces. Langmuir, 23: 10235-43.

[28] Sekiguchi, Y., Takahashi, K., \& Sato, C., 2015, Adhesion mechanism of a gecko-inspired oblique structure with an adhesive tip for asymmetric detachment. Journal of Physics D: Applied Physics, 48(47), 475301.

[29] Autumn, K., Peattie, A.M., 2002, Mechanisms of Adhesion in Geckos. Int Comp Biol 42, 1081-1090.

[30] Autumn, K., Hsieh, S.T., Dudek, D.M., Chen, J., Chitaphan, C., Full, R.J., 2006, Dynamics of geckos running vertically. J Exp Biol 209, 260-272. 
[31] Huber, G., Mantz, H., Spolenak, R., Mecke, K., Jacobs, K., Gorb, S. N. and Arzt, E., 2005, Evidence for capillarity contributions to gecko adhesion from single spatula nanomechanical measurements, Proc. Natl. Acad. Sci. USA, 102(45), 16293-16296.

[32] Ozer, A., 2016, Mechanics of bio-inspired attachment systems contacting with rough surface: effect of spring orientation angle on hierarchical spring model. Journal of Adhesion Science and Technology, 1-15.

[33] O'Rorke, R. D., Steele, T. W. J., \& Taylor, H. K., 2016, Bioinspired fibrillar adhesives: a review of analytical models and experimental evidence for adhesion enhancement by surface patterns. Journal of Adhesion Science and Technology, 30(4), 362-391.

[34] Varenberg, M., \& Gorb, S. (2007). Shearing of fibrillar adhesive microstructure: friction and shear-related changes in pull-off force. Journal of The Royal Society Interface, 4(15), 721-725.

[35] Persson, B. N. J. 2002 Adhesion between elastic bodies with randomly rough surfaces. Phys. Rev. Lett. 89, 245502.

[36] Guduru P.R., 2007, Detachment of a rigid solid from an elastic wavy surface: Theory, Journal of the Mechanics and Physics of Solids 55: 445-472.

[37] Carbone G., Mangialardi L., 2004, Adhesion and friction of an elastic half-space in contact with a slightly wavy rigid surface, Journal of the Mechanics and Physics of Solids, 52 (6), $1267-1287$.

[38] Wu J-J., 2012, Numerical simulation of the adhesive contact between a slightly wavy surface and a half-space, Journal of Adhesion Science and Technology, 26, 331-351.

[39] G. Carbone, E. Pierro and G. Recchia, Loading-unloading hysteresis loop of randomly rough adhesive contacts, 2016, Physiscal Review E, 92, 062404.

[40] M. Ciavarella, A. Papangelo, L. Afferrante, 2017, Adhesion between self-affine rough surfaces: Possible large effects in small deviations from the nominally Gaussian case, Tribology International, 109, 435-440.

[41] Guduru P.R. and Bull C., 2007, Detachment of a Rigid Solid from a Wavy Elastic Surface Experiments, Journal of the Mechanics and Physics of Solids 55: 473-488.

[42] Martina D., Creton C., Damman P., et al., 2012, Adhesion of soft viscoelastic adhesives on periodic rough surfaces, Soft Matter 8 (19), 5350-5357, doi: 10.1039/c2sm07059f.

[43] Martina, D., Creton C., Damman P., Jeusette M. \& Lindner A. 2012 Adhesion of soft viscoelastic adhesives on periodic rough surfaces, Soft MAtter, 8, 5350.

[44] Dies, L., Restagno, F., Weil, R., Leger, L. \& Poulard C., 2015, Role of adhesion between asperities in the formation of elastic solid/solid contacts, Eur. Phys. J. E, 38, 130.

[45] Arzt, E., Gorb, S., and Spolenak, R., 2003, From micro to nano contacts in biological attachment devices. Proc. Natl. Acad. Sci. U. S. A., 100(19), 10603-10606.

[46] Tian, Y., Pesika, N., Zeng, H.B., Rosenberg, K., Zhao, B.X., McGuiggan, P., Autumn, K. and Israelachvili, J., 2006, Adhesion and friction in gecko toe attachment and detachment, Proc. Natl. Acad. Sci. USA, 103, 19320-19325.

[47] Autumn, K., Dittmore, A., Santos, D., Spenko, M. and Cutkosky, M., 2006, Frictional adhesion: a new angle on gecko attachment, The Journal of Experimental Biology, 209, 3569-3579.

[48] Lepore E., Pugno F. and Pugno N. M., 2012, Optimal Angles for Maximal Adhesion in Living Tokay Geckos, The Journal of Adhesion, 88:10, 820-830.

[49] Kaelble, D. H. (1959). Theory and analysis of peel adhesion: mechanisms and mechanics. Transactions of the Society of Rheology, 3(1), 161-180.

[50] Kaelble, D. H. (1965). Peel Adhesion: Micro-Fracture Mechanics of Interfacial Unbonding of Polymers. Transactions of the Society of Rheology, 9(2), 135-163. 
[51] Kendall, K., 1975, Thin-film peeling-the elastic term, J. Phys. D: Appl Phys 8, 1449-1452.

[52] Barquins, M. and Ciccotti, M., 1997, On the kinetics of peeling of an adhesive tape under a constant imposed load, Int. J. Adhesion and Adhesives, 17, 65-68.

[53] ISO 8510-1 (1990) Adhesives - peel test for a flexible-bonded-to-rigid test specimen assembly - part 1: 90 degree peel.

[54] ISO 8510-2 (2006) Adhesives - peel test for a flexible-bonded-to-rigid test specimen assembly - part 2: 180 degree peel.

[55] Creton, C., \& Ciccotti, M. (2016). Fracture and adhesion of soft materials: a review. Reports on Progress in Physics, 79(4), 046601.

[56] Villey, R., Creton, C., Cortet, P. P., Dalbe, M. J., Jet, T., Saintyves, B., ... \& Ciccotti, M. (2015). Rate-dependent elastic hysteresis during the peeling of pressure sensitive adhesives. Soft Matter, 11(17), 3480-3491.

[57] Villey, R., Cortet, P.P., Creton, C., Ciccotti, M., 2017, In-situ measurement of the large strain response of the fibrillar debonding region during the steady peeling of pressure sensitive adhesives, Int J Fract., 204,175-190.

[58] Callies, X., Herscher, O., Fonteneau, C., Robert, A., Pensec, S., Bouteiller, L., ... \& Creton, C. (2016). Combined Effect of Chain Extension and Supramolecular Interactions on Rheological and Adhesive Properties of Acrylic Pressure-Sensitive Adhesives. ACS applied materials \& interfaces, 8(48), 33307-33315.

[59] Creton, C., \& Leibler, L. (1996). How does tack depend on contact time and contact pressure. J. Polym. Sci. B, 34, 545-554.

[60] Hui, C. Y., Lin, Y. Y., \& Baney, J. M. (2000). The mechanics of tack: viscoelastic contact on a rough surface. Journal of Polymer Science Part B: Polymer Physics, 38(11), 1485-1495.

[61] Persson, B. N. J., Albohr, O., Creton, C., \& Peveri, V. (2004). Contact area between a viscoelastic solid and a hard, randomly rough, substrate. The Journal of chemical physics, 120(18), 8779-8793.

[62] Lakrout, H., Sergot, P., \& Creton, C. (1999). Direct observation of cavitation and fibrillation in a probe tack experiment on model acrylic pressure-sensitive-adhesives. The Journal of Adhesion, 69(3-4), 307-359.

[63] Kwak, M. K., Jeong, H.-E., Suh, K. Y. 2011 Rational design and enhanced biocompatibility of a dry adhesive medical skin patch. Adv. Mater. 23, 3949-3953.

[64] Chivers, R. A., 2001, Easy removal of pressure sensitive adhesives for skin applications, Int. J. Adhesion \& Adhesives, 21, 381-388.

[65] Pereira, J. M., Mansour, J. M. andDavis B. R., 1991, Dynamic measurement of the viscoelastic properties of skin, Journal of biomechanics, 24(2), 157-162.

[66] Edwards, C. and Marks, R., 1995, Evaluation of biomechanical properties of human skin, Clinics in dermatology, 13(4), 375-380.

[67] Silver, F. H., Freeman, J. W. and CeVoe, D., 2001, Viscoelastic properties of human skin and processed dermis, Skin Research and Technology, 7, 18-23.

[68] Boyer, G., Lyon, C. N. R. S., Zahouani, H., Le Bot, A. and Laquieze, L., 2007, In vivo characterization of viscoelastic properties of human skin using dynamic micro-indentation, Engineering in Medicine and Biology Society, 2007. EMBS 2007. 29th Annual International Conference of the IEEE.

[69] Menga N, Putignano C, Carbone G, Demelio GP. 2014 The sliding contact of a rigid wavy surface with a viscoelastic half-space. Proc. R. Soc. A, 470, 20140392, 2014.

[70] Menga N, Afferrante L, Carbone G. 2016 Effect of thickness and boundary conditions on the 
behavior of viscoelastic layers in sliding contact with wavy profiles. Journal of the Mechanics and Physics of Solids. In press. Doi: 10.1016/j.jmps.2016.06.009

[71] Menga, N., Foti, D., \& Carbone, G. Viscoelastic frictional properties of rubber-layer roller bearings (RLRB) seismic isolators. Meccanica, 1-11.

[72] Bosia, F., Colella, S., Mattoli, V., Mazzolai, B., \& Pugno, N. M. (2014). Hierarchical multiple peeling simulations. RSC Advances, 4(48), 25447-25452.

[73] Wolff, J. O., Grawe, I., Wirth, M., Karstedt, A., \& Gorb, S. N. (2015). Spider's super-glue: thread anchors are composite adhesives with synergistic hierarchical organization. Soft Matter, 11(12), 2394-2403.

[74] Afferrante L. and Carbone G., 2016, The ultratough peeling of elastic tapes from viscoelastic substrates, Journal of the Mechanics and Physics of Solids, 96, 223-234.

[75] Kaelble, D.H., 1960, Theory and analysis of peel adhesion: bond stresses and distributions, Trans. Soc. Rheol. 4, 45-73.

[76] Johnson, K.L., Kendall, K., Roberts, A.D., 1971, Surface energy and the contact of elastic solids, Proc. R. Soc. London A, 324, 301-313.

[77] Lin, Y-Y., Chen, H-Y., 2006, Effect of Large Deformation and Material Nonlinearity on the JKR (Johnson-Kendall-Roberts) Test of Soft Elastic Materials, Journal of Polymer Science: Part B: Polymer Physics, DOI: 10.1002/polb.20914.

[78] Maugis, D. and Barquins, M., in Adhesion 12, Elsevier ASP, London, 1988, 205-222.

[79] Ciccotti, M. Giorgini, B., Vallet, D. and Barquins, M., 2004, Complex dynamics in the peeling of an adhesive tape, Int. J Adhesion and Adhesives, 24, 143-151.

[80] Rumi, De and Ananthakrishna, G., 2006, Dynamics of the peel front and the nature of acoustic emission during peeling of an adhesive tape, Physical Review Letters, 97(16), 165503.

[81] Dalbe, M.-J., Santucci, S., Cortet, P.-P. and Vanel, L., 2014, Strong dynamical effects during stick-slip adhesive peeling, Soft Matter, 10(1), 132-138.

[82] Dalbe, M.-J., Santucci, S.,Vanel, L. and Cortet, P.-P., 2014, Peeling-angle dependence of the stick-slip instability during adhesive tape peeling, Soft Matter, 10(48), 9637-9643.

[83] Afferrante, L., Carbone, G., Demelio, G. and Pugno N., 2013, Adhesion of elastic thin films: Double peeling of tapes versus axisymmetric peeling of membranes, Tribol. Lett., 52, 439-447.

[84] Putignano C., Afferrante L., Mangialardi L. and Carbone G., 2014, Equilibrium states and stability of pre-tensioned adhesive tapes, Beilstein J Nanotechnol., 5, 1725-1731.

[85] Abramowitz, M. and Stegun, I. A. (Eds.). "Clausen's Integral and Related Summations" §27.8 in Handbook of Mathematical Functions with Formulas, Graphs, and Mathematical Tables, 9th printing. New York: Dover, pp. 1005-1006, 1972. 